\title{
INTRA-CANALICULAR GELATIN IMPLANTS IN THE TREATMENT OF KERATO-CONJUNCTIVITIS SICCA*
}

\author{
BY \\ WALLACE S. FOULDS \\ Addenbrooke's Hospital, Cambridge
}

IN patients suffering from kerato-conjunctivitis sicca, occlusion of the lacrimal puncta may be followed by a marked relief of symptoms and an improvement in the appearance of the cornea and conjunctiva. Unfortunately, this is not always the case, for some patients are no better after this procedure and a few may actually develop epiphora.

Stallard (1958) has stressed how thorough cautery to the canaliculi has to be if effective occlusion is to be attained, and there is little doubt that the resulting obliteration of the canaliculi is permanent. Because of this, many surgeons are reluctant to use the procedure, reserving it for severe and longstanding cases of kerato-conjunctivitis sicca where they can be fairly certain that undesirable sequelae are unlikely to be encountered.

It is probably true that cases of kerato-conjunctivitis sicca are more common than is generally realized, and that many of these might be helped by occlusion of the puncta if it could be ascertained beforehand that this procedure would, in fact, relieve the patient's symptoms and not be followed by epiphora. With this in view a simple method of creating a temporary obstruction of the canaliculi by the insertion of fine water-soluble gelatin rods has been developed and has proved of value in assessing the probable effects of permanent obstruction of the canaliculi.

\section{Material}

Pure powdered gelatin to which a small quantity of distilled water $(1 \mathrm{ml} . / \mathrm{g}$. $)$ has been added is heated in a water bath until the gelatin dissolves and a thick gel results. By dipping a cold glass rod into this gel and withdrawing it, fine solid rods of gelatin are easily formed, and with practice, fairly uniform lengths of approximately $0.5 \mathrm{~mm}$. in diameter can easily be made (Fig. 1). These rods are cut into 4-cm. lengths and stored in closely-stoppered glass containers.

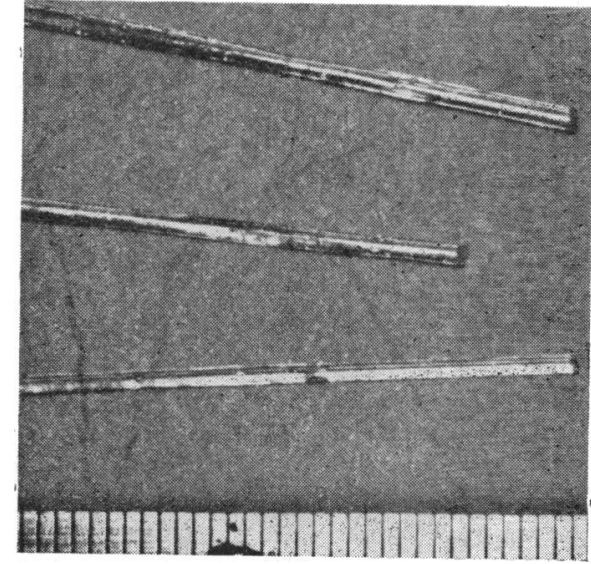

Fig. 1.-Gelatin rods used to occlude the lacrimal puncta. Scale divisions represent $0.5 \mathrm{~mm}$ 


\section{Method}

Two drops of amethocaine 1 per cent. are instilled into the eye to be treated. The lacrimal punctum is dilated with a Nettleship's dilator and a gelatin rod is inserted in one movement so as to fill the whole length of the canaliculus. (Fig. 2). The excess rod is divided with scissors at the punctum. The short projecting end of this rod very quickly becomes rounded and smooth when it comes in contact

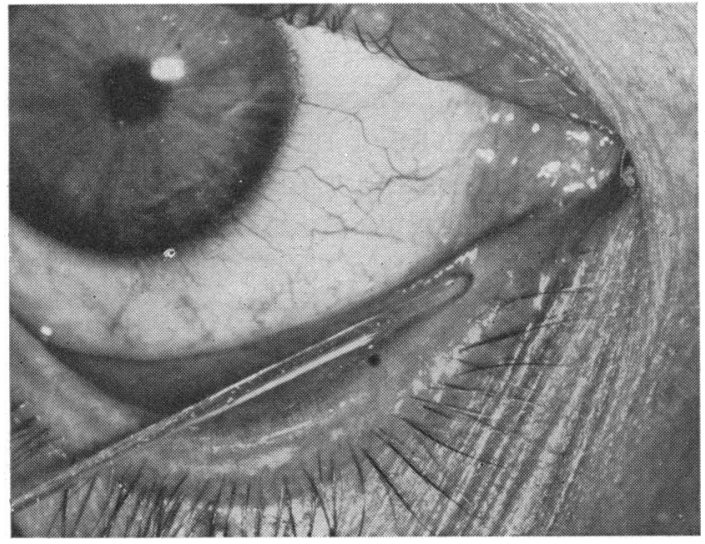
with the moist bulbar conjunctiva. It is important to slide the rod straight into the canaliculus as it soon becomes adherent and it may be difficult subsequently to ensure that a suitable length of gelatin remains within the canaliculus.

Fig. 2.- Insertion of a gelatin rod into the lower lacrimal punctum.

\section{Results}

At the time of writing the procedure has been used in thirty patients, and in none has there been any adverse reaction to the presence of the implants. A few patients have noted slight transient discomfort immediately following the insertion of the rods but this has not lasted long. No local inflammatory reaction to the presence of the gelatin within the canaliculi has been found in any of the patients.

In the series of patients treated, the clinical picture varied from classical cases of Sjögren's syndrome (Sjögren, 1933), with marked filamentary keratitis, typical staining of the conjunctiva with Rose Bengal, and dry conjunctival sacs as measured by Schirmer's test (Schirmer, 1903), to cases in which the diagnosis of kerato-conjunctivitis sicca was in some doubt, there being chronic conjunctival hyperaemia accompanied by a superficial epithelial keratitis or an abnormal pre-corneal tear film and a reduced secretion of tears to Schirmer's test.

As would be expected from such a diverse group of patients, the response to the implants varied greatly. In some there was a dramatic improvement in symptoms lasting for periods of from 4 days to 2 weeks, after which time it could be shown that the implants had dissolved and the patency of the canaliculi had been restored. Such cases were considered suitable for permanent surgical occlusion of the puncta. Others either experienced no symptomatic relief from the procedure or even developed temporary epiphora. Such cases were obviously not suitable for cautery to the puncta. 
It is to be noted that, because the reaction to the presence of the rods within the canaliculi is as a rule so slight, there is no reflex stimulation of tear secretion and absence of epiphora under these conditions represents the state of affairs attained some weeks after cautery to the puncta. In the days immediately following cauterization of the puncta there is inevitably a reflex stimulation of tear flow which may result in a temporary epiphora until the inflammatory reaction has settled down. Thus, in three cases in which the insertion of gelatin rods was followed by a dramatic improvement in symptoms without noticeable epiphora, cautery to the puncta not only produced the desired symptomatic relief but in addition resulted in epiphora which lasted until the cauterized areas had completely healed. When healing had taken place the epiphora ceased and the relief of symptoms was so marked that in each case the other eye was similarly treated.

In some cases in which the diagnosis of kerato-conjunctivitis sicca was not certain, the tear-flow caused a rapid dissolving of the implants. These patients noted a clear sticky discharge from the eye for 24 hours after the insertion of the implants.

It is interesting that, in the patients who developed a temporary epiphora after the insertion of the rods, Schirmer's test before their insertion showed a grossly deficient tear secretion even after stimulation of the nasal mucosa with a cotton-wool swab. In such cases cautery to the puncta would undoubtedly have been followed by intractable epiphora. The mere finding of a reduced tear-flow is no guarantee that the case is suitable for occlusion of the puncta.

While it may be true that the insertion of gelatin rods into the canaliculi of these patients was a stronger stimulus to tear production than nasal irritation, it is also possible that much of the tear-flow was escaping by way of the puncta before it could be absorbed by the Schirmer's test paper, so giving an erroneously low estimate of the tear secretion.

\section{Summary}

A method of creating a temporary obstruction of the canaliculi by the insertion of water-soluble gelatin rods is described. It has proved valuable in the diagnosis of kerato-conjunctivitis sicca and in assessing the symptomatic improvement to be expected by permanent occlusion of the puncta.

I am indebted to Mr. Barrie Jones of the Institute of Ophthalmology, London, for]his encouragement and his help in assessing the effect of the implants on several patients under his care. I also wish to thank the staff of the Pharmaceutical Department of Addenbrooke's Hospital for their help in the preparation of the implants and Mr. Vince of the Photographic Department of the same Hospital for the illustrations.

\section{REFERENCES}

SCHIRMER, O. (1903). v. Graefes Arch. Ophthal., 56, 197.

SJögren, H. (1933). Acta ophthal. (Kbh.), Suppl. 2.

STAllard, H. B. (1958). "Eye Surgery", 3rd ed., p. 303. Wright, Bristol. 